\title{
Mourre estimates for a 2D magnetic quantum Hamiltonian on strip-like domains
}

\author{
Philippe Briet ${ }^{1}$, Peter D. Hislop ${ }^{2}$, Georgi Raikov $^{3}$, Eric Soccorsi ${ }^{1}$
}

April 7, 2009

${ }^{1}$ Centre de Physique Théorique de Marseille UMR 6207 - Unité Mixte de Recherche du CNRS et des Universités Aix-Marseille I, Aix-Marseille II et de l' Université du Sud Toulon-Var, Laboratoire affilié à la FRUMAM

E-mail: briet,soccorsi@cpt.univ-mrs.fr

${ }^{3}$ Department of Mathematics, University of Kentucky, Lexington, KY 40506-0027, USA

E-mail: hislop@ms.uky.edu

${ }^{3}$ Facultad de Matemáticas, Pontificia Universidad Católica de Chile, Av. Vicuña Mackenna 4860, Santiago de Chile

E-mail: graikov@mat.puc.cl

\begin{abstract}
We consider a 2D Schrödinger operator $H_{0}$ with constant magnetic field defined on a strip of finite width. The spectrum of $H_{0}$ is absolutely continuous and contains a discrete set of thresholds. We perturb $H_{0}$ by an electric potential $V$, and establish a Mourre estimate for $H=H_{0}+V$ when $V$ is periodic in the infinite direction of the strip, or decays in a suitable sense at infinity. In the periodic case, for each compact subinterval $I$ contained in between two consecutive thresholds, we show as a corollary that the spectrum of $H$ remains absolutely continuous in $I$, provided the period and the size of the perturbation are sufficiently small. In the second case we obtain that the singular continuous spectrum of $H$ is empty, and any compact subset of the complement of the thresholds set contains at most a finite number of eigenvalues of $H$, each of them having finite multiplicity. Moreover these Mourre estimates together with some of their spectral consequences generalize to the case of $2 \mathrm{D}$ magnetic Schrödinger operators defined on $\mathbb{R}^{2}$ for suitable confining potentials modeling Dirichlet boundary conditions.
\end{abstract}

AMS 2000 Mathematics Subject Classification: 35J10, 81Q10, 35P20

Keywords: Schrödinger operators, constant magnetic field, Mourre estimate, periodic potential.

\section{Introduction and contents}

1.1. The study of the quantum motion of a charged particle in a two-dimensional medium submitted to an orthogonal magnetic field of constant strength is at the center of theoretical explanation of edge currents in Hall systems, and is a source of interesting spectral problems. Some of them have been rigourously investigated by many authors in recent years $([13,6,20,12,8,4,9,10,11,2,15,16])$. 
Edge currents have some connection with the integer quantum Hall effect. References to mathematical papers describing the relationship between edge currents and the integer quantum Hall effect can be found in $[1,19,7,3]$. The quantum devices studied with regard to the integer quantum Hall effect are distinguished by the fact that there is at least one edge. The existence of an edge profoundly changes the transport and spectral properties of the quantum system. A state $\psi$ carries an edge current if the expectation of the velocity operator along the edge in the state $\psi$ is non vanishing. Such a state is spatially concentrated near the edge (see $[6,9,10,4,15,16]$ ) and is thus called an edge state.

If the particle is constrained to a semi-infinite system like a half-plane, the existence of edge currents can be shown through positive commutator estimates $([6,12])$. In this case, for an infinite system in the $y$-direction, $y$ is a bona-fide conjugate operator for the magnetic operator $H$ under study in the sense of Mourre, since the commutator $[H, i y]$ is proportional to the velocity operator in the $y$-direction along the edge, and the double commutator $\left[\left[H_{0}, y\right], y\right]=2 i$. Thus for these one-edge geometries the existence of edge currents is equivalent to the existence of intervals of absolutely continuous spectrum for the corresponding Hamiltonian (see [6, 20, 12, 4, 15]).

This need not be the case, however, for more complicated edge geometries. For those situations, there may be edge currents but the spectrum need not be absolutely continuous (cf. [8, 9, 10, 11, 4, 16]). For instance, in the case of a strip of finite width, adding a second edge radically changes the picture observed for one-edge geometries, since the Hall current has different signs on opposite edges. Hence Mourre theory of positive commutators does not apply with $y$ as a conjugate operator. Actually, for these models, the existence of edge currents does not generally have any implication for the spectral type of the operator. Indeed edge currents exist for the two-edge cylinder geometry, even though the Hamiltonian has purely discrete spectrum (cf. $[9,11,16]$ ).

For an infinite strip, however, the presence of edge currents can be spectrally translated as the existence of intervals of absolutely continuous spectrum for $H$. This can be achieved by proving Mourre estimates for appropriate conjugate operators (see $[4,2,16]$ ). One of the benefits of a local positive commutator of this type is its stability under perturbation. It is therefore particularly useful to prove the persistence of edge currents in presence of weak disorder (cf. $[8,4,16]$ ).

1.2. In this short paper based on the articles [2] and [16], we consider a $2 \mathrm{D}$ Schrödinger operator $H_{0}$ with constant magnetic field defined on an infinite strip of finite width. We impose Dirichlet boundary conditions on both edges of the strip. Using the invariance of the system in the infinite direction $y$ we prove that $H_{0}$ is unitarily equivalent to an analytically fibered operator with real analytic band functions $\left\{E_{j}\right\}_{j=1}^{+\infty}$ such that $k E_{j}^{\prime}(k)>0$ for all $k \in \mathbb{R}$. Thus the spectrum of $H_{0}$ is absolutely continuous and contains a discrete set of thresholds $\left\{E_{j}(0)\right\}_{j=1}^{+\infty}$. Further, from the monotonicity property of the band functions, we define a family of conjugate operators $A_{f}$, where $f$ is taken in some subset of $C^{\infty}(\mathbb{R})$, involving a Mourre estimate for $H_{0}$. Then we perturb $H_{0}$ by an electric potential $V$ and, using the stability of local positive commutator $\left[H_{0}, A_{f}\right]$ under perturbation, establish a Mourre estimate for $H=H_{0}+V$ when 1) $V$ is periodic in the infinite direction of the strip, or 2) $V$ decays in a suitable sense at infinity. In both cases we give an explicit expression, adapted to the perturbation $V$ under consideration, of the conjugate operator $A_{f}$ involved in the corresponding positive commutator estimate. Then using Mourre theory (see [21,5]), we derive some useful spectral information on the system. In the periodic case, when the period and the size of $V$ are taken sufficiently small, we obtain that the spectrum of $H$ remains absolutely continuous in any compact subinterval contained in between two consecutive thresholds. In the second case we show that the singular continuous spectrum of $H$ is empty, while any compact subset of the complement of the thresholds set contains at most a finite number of eigenvalues of $H$, each of them having finite multiplicity. As a conclusive remark we point out that these Mourre estimates together with some of their spectral consequences generalize to the case of $2 \mathrm{D}$ magnetic Schrödinger operators defined on $\mathbb{R}^{2}$ for suitable confining potentials modeling Dirichlet boundary conditions. 
1.3. The paper is organized as follows. In Section 2 we describe the system under consideration. More precisely, we introduce in Subsection 2.1 the main notations used throughout the article. In Subsection 2.2 , we define the operator $H_{0}$ under study and recall some of its basic spectral properties. Section 3 contains Mourre estimates for $H_{0}+V$ and suitable perturbations $V$. In Subsection 3.2 we define a family of conjugate operators for $H_{0}$. In Subsection 3.3 we address the case of a periodic potential, while Subsection 3.4 is devoted to decreasing perturbations. In Section 4 we extend some of the results and techniques used in Section 3 to the case of magnetic Schrödinger operators defined in $\mathbb{R}^{2}$.

This is a review article so most of the techniques presented in this paper have already been published in $[2,16]$, sometimes in a slightly different form. Here we present a unified version of the ideas used in these two articles to establish Mourre estimates for 2D magnetic Schrödinger operators defined on strip-like domains. More precisely, the statements of Subsection 3.4 have already been given in [2]. Those of Subsection 3.3 are inspired from the results obtained in [16] for the model defined in Section 4, while Proposition 4.1 and Theorem 4.1 in Section 4 extend and complete Proposition 1.1 of [16].

\section{Definitions and main notations}

2.1. In this subsection we introduce some basic notations used throughout the article. Let $X_{1}, X_{2}$ be two separable Hilbert spaces. We denote by $\mathcal{B}\left(X_{1}, X_{2}\right)$ (resp., by $S_{\infty}\left(X_{1}, X_{2}\right)$ ) the class of bounded (resp., compact) operators $T: X_{1} \rightarrow X_{2}$. If $X_{1}=X_{2}=X$ we write $\mathcal{B}(X)$ or $S_{\infty}(X)$ instead of $\mathcal{B}(X, X)$ or $S_{\infty}(X, X)$. Also, if the indication of the Hilbert space(s) where the corresponding operators act is irrelevant, we omit it in the notations of the classes $\mathcal{B}$ and $S_{\infty}$.

Let $T=T^{*}$. We denote by $\mathbb{P}_{\mathcal{O}}(T)$ the spectral projection of $T$ associated with the Borel set $\mathcal{O} \subset \mathbb{R}$. Finally, we write $p_{x}$ and $p_{y}$ for $-i \frac{\partial}{\partial x}$ and $-i \frac{\partial}{\partial y}$ respectively.

2.2. In this subsection we define the operator $H_{0}$ and collect some of its spectral properties that will be needed in the sequel. For $L>0$ set $I_{L}=(-L, L), \mathcal{S}_{L}=I_{L} \times \mathbb{R}$ and consider the 2D Schrödinger operator with constant scalar magnetic field $b>0$

$$
H_{0}:=p_{x}^{2}+\left(p_{y}-b x\right)^{2}
$$

defined on $\left\{u \in \mathrm{H}^{2}\left(\mathcal{S}_{L}\right) \mid u_{\mid \partial \mathcal{S}_{L}}=0\right\}$ where $\mathrm{H}^{2}\left(\mathcal{S}_{L}\right)$ denotes the second-order Sobolev space on $\mathcal{S}_{L}$. Then we have

$$
\mathcal{F} H_{0} \mathcal{F}^{*}=\int_{\mathbb{R}}^{\oplus} \hat{H}_{0}(k) d k,
$$

where $\mathcal{F}$ is the partial Fourier transform with respect to $y$, and

$$
\hat{H}_{0}(k):=p_{x}^{2}+(k-b x)^{2}, \quad k \in \mathbb{R},
$$

is the operator defined on $D\left(\hat{H}_{0}\right):=\left\{w \in \mathrm{H}^{2}\left(I_{L}\right) \mid w(-L)=w(L)=0\right\}$. The spectrum $\sigma\left(\hat{H}_{0}(k)\right)$ of the operator $\hat{H}_{0}(k), k \in \mathbb{R}$, is discrete and simple. We note $\left\{E_{j}(k)\right\}_{j=1}^{\infty}$ the increasing sequence of the eigenvalues of $\hat{H}_{0}(k)$. They are even real analytic functions of $k \in \mathbb{R}$ (see [18]), and the minimax principle implies

$$
E_{j}(k)=k^{2}(1+o(1)), \quad k \rightarrow \pm \infty, j \in \mathbb{N}:=\{1,2, \ldots\} .
$$

Moreover, by [13, Theorem 2] we have

$$
k E_{j}^{\prime}(k)>0, \quad k \neq 0, j \in \mathbb{N} .
$$

Thus $\sigma\left(H_{0}\right)=\sigma_{\mathrm{ac}}\left(H_{0}\right)=\left[E_{1}(0), \infty\right)$, where $\sigma_{\mathrm{ac}}\left(H_{0}\right)$ stands for absolutely continuous spectrum of $H_{0}$, and $\mathcal{E}_{j}:=E_{j}(0), j \in \mathbb{N}$, are thresholds in $\sigma\left(H_{0}\right)$. We set $\mathcal{Z}:=\bigcup_{j \in \mathbb{N}}\left\{\mathcal{E}_{j}\right\}$.

Finally, each $E_{r}, r \in \mathbb{N}$, being a continuous increasing function in $(0,+\infty)$, we have the following 
Lemma 2.1. ([2, Lemma 3.1]) Let $n \in \mathbb{N}, E \in\left(\mathcal{E}_{n}, \mathcal{E}_{n+1}\right)$. Then there exists $\delta_{0}=\delta_{0}(E) \in$ $(0, \operatorname{dist}(E, \mathcal{Z}))$ such that the interval $\Delta_{E}=\left[E-\delta_{0}, E+\delta_{0}\right]$ satisfies

$$
E_{r}^{-1}\left(\Delta_{E}\right)=\emptyset, \quad r \geq n+1,
$$

and, if $n \geq 2$,

$$
E_{r}^{-1}\left(\Delta_{E}\right) \cap E_{s}^{-1}\left(\Delta_{E}\right)=\emptyset, \quad r \neq s, \quad r, s=1, \ldots, n
$$

\section{Mourre estimates}

3.1. We now examine the spectral properties of the Hamiltonian $H=H_{0}+V$ for suitable perturbations $V$. We use the commutator method of Mourre [21, 5] (see also [14]). For the strip geometry, (2.3) shows that the derivative $E_{j}^{\prime}, j \in \mathbb{N}$, does not have a fixed sign. Consequently the local commutator used for the one-edge geometries in $[6,12,15]$ does not immediately apply. We preliminarily construct a class of appropriate conjugate operators involving a Mourre estimate for $H_{0}$ on suitable subintervals of $\left(\mathcal{E}_{n}, \mathcal{E}_{n+1}\right), n \in \mathbb{N}$. The advantage of this Mourre estimate is its stability under specific perturbations $V$, and the spectral consequences that can be derived from it. Namely if $V$ is $T$-periodic we show in Section 3.3 that the absolutely spectrum of $H$ persists on any subinterval of $\left(\mathcal{E}_{n}, \mathcal{E}_{n+1}\right), n \in \mathbb{N}$, for $\|V\|_{\infty}$ and $T$ sufficiently small. These results are similar to those of [8] obtained for a shifted harmonic oscillator. Finally if $V$ is $H_{0}$-compact and satisfies some additional technical conditions, we obtain in Section 3.4 that the singular continuous spectrum of $H$ is empty.

For $\gamma>0$ we define $\mathcal{H}_{\gamma}:=D\left(H_{0}^{\gamma / 2}\right)$ as a Hilbert space equipped with the scalar product $\left\langle H_{0}^{\gamma / 2} u, H_{0}^{\gamma / 2} v\right\rangle_{L^{2}\left(\mathcal{S}_{L}\right)}, u, v \in D\left(H_{0}^{\gamma / 2}\right)$, and denote by $\mathcal{H}_{-\gamma}$, the closure of $L^{2}\left(\mathcal{S}_{L}\right)$ in the norm $\left\|H_{0}^{-\gamma / 2} u\right\|_{L^{2}\left(\mathcal{S}_{L}\right)}, u \in L^{2}\left(\mathcal{S}_{L}\right)$.

3.2. In this subsection we define a family of conjugate operators for $H_{0}$. To this purpose we consider the set $\mathcal{M}$ of infinitely differentiable functions in $\mathbb{R}$ which together with all its derivatives grow no faster than polynomials. For $f \in \mathcal{M}$ we introduce the operator

$$
A_{f}=A_{f}^{*}=\frac{1}{2}\left(y f\left(p_{y}\right)+\bar{f}\left(p_{y}\right) y\right)
$$

originally defined in $C_{0}^{\infty}\left(\mathbb{R}_{y}, D\left(\hat{H}_{0}\right)\right)$ and then closed in $L^{2}\left(\mathcal{S}_{L}\right)$. Note that $C_{0}^{\infty}\left(\mathbb{R}_{y}, D\left(\hat{H}_{0}\right)\right)$ is dense in $\mathcal{H}_{2}$ hence $D\left(A_{f}\right) \cap \mathcal{H}_{2}$ is dense in $\mathcal{H}_{2}$.

Lemma 3.1. ([2, Proposition 3.1]) Let $n \in \mathbb{N}, E \in\left(\mathcal{E}_{n}, \mathcal{E}_{n+1}\right)$. Assume that $\delta_{0} \in(0, \operatorname{dist}(E, \mathcal{Z}))$ is chosen to satisfy (2.4) and (2.5) according to Lemma 2.1. Let $\chi \in C_{0}^{\infty}(\mathbb{R})$, supp $\chi=\left[E-\delta_{0}, E+\delta_{0}\right]$ and choose $f \in \mathcal{M}$ such that

$$
C_{E}\left(\delta_{0}\right):=\min _{r=1, \ldots, n} \inf _{k \in E_{r}^{-1}\left(\left[E-\delta_{0}, E+\delta_{0}\right]\right)} \operatorname{Re}(f(k)) E_{r}^{\prime}(k)>0 .
$$

Then we have

$$
\chi\left(H_{0}\right)\left[H_{0}, i A_{f}\right] \chi\left(H_{0}\right) \geq C_{E}\left(\delta_{0}\right) \chi\left(H_{0}\right)^{2},
$$

as a quadratic form on $D\left(A_{f}\right) \cap \mathcal{H}_{2}$.

Notice from (2.3) that there exists $f \in \mathcal{M}$ satisfying (3.2).

Proof. For $\chi \in C_{0}^{\infty}(\mathbb{R})$ we have

$$
\chi\left(H_{0}\right)\left[H_{0}, i A_{f}\right] \chi\left(H_{0}\right)=2 \mathcal{F}^{*}\left(\sum_{r, s=1}^{\infty} \int_{\mathbb{R}}^{\oplus} \chi\left(E_{r}(k)\right) \chi\left(E_{s}(k)\right) \operatorname{Re}(f(k)) p_{r}(k)(k-b x) p_{s}(k) d k\right) \mathcal{F},
$$


where

$$
p_{r}(k):=\left\langle\cdot, \psi_{r}(\cdot ; k)\right\rangle \psi_{r}(\cdot ; k), k \in \mathbb{R}, r \in \mathbb{N},
$$

$\psi_{r}(\cdot ; k): I_{L} \rightarrow \mathbb{R}$ being the real-valued normalized in $L^{2}\left(I_{L}\right)$ eigenfunction of the operator $\hat{H}_{0}(k)$ corresponding to the eigenvalue $E_{j}(k)$.

Using (2.4) and (2.5), we find that (3.4) reduces to

$$
\chi\left(H_{0}\right)\left[H_{0}, i A_{f}\right] \chi\left(H_{0}\right)=2 \mathcal{F}^{*}\left(\sum_{r=1}^{n} \int_{\mathbb{R}}^{\oplus} \chi\left(E_{r}(k)\right)^{2} \operatorname{Re}(f(k))\left\langle(k-b x) \psi_{r}(k), \psi_{r}(k)\right\rangle p_{r}(k) d k\right) \mathcal{F} .
$$

This, combined with the Feynman-Hellmann formula

$$
E_{r}^{\prime}(k)=2\left\langle(k-b x) \psi_{r}(k), \psi_{r}(k)\right\rangle,
$$

yields

$$
\chi\left(H_{0}\right)\left[H_{0}, i A_{f}\right] \chi\left(H_{0}\right)=\mathcal{F}^{*}\left(\sum_{r=1}^{n} \int_{\mathbb{R}}^{\oplus} \operatorname{Re}(f(k)) E_{r}^{\prime}(k) \chi\left(E_{r}(k)\right)^{2} p_{r}(k) d k\right) \mathcal{F},
$$

so (3.3) follows from this and (3.2).

Remark 3.1. Notice from the computations made in the proof of Lemma 3.1 that

$$
\left[H_{0}, i A_{f}\right]=2 \operatorname{Re}\left(f\left(p_{y}\right)\right) v_{y},
$$

where $v_{y}:=p_{y}-b x$ is the velocity operator in the $y$-direction along the edges.

The benefit of a local positive commutator of the type of (3.3) is its stability under sufficiently small perturbations. Nevertheless the commutator $\left[V, i A_{f}\right]$ is generally unbounded unless $V$ is $y$-independent. Hence a Mourre inequality for $H=H_{0}+V$ requires control in terms of the $\left[H_{0}, i A\right]$ as in Section 3.4, except for some specific cases, as perturbations periodic in the $y$-direction.

3.3. First we address the case of a bounded $y$-periodic potential $V$ with period $T>0$ :

$$
V(x, y+T)=V(x, y),(x, y) \in \mathcal{S}_{L} .
$$

Following the idea of [16, Section 1.3], let $U_{\alpha}=e^{i \alpha p_{y}}, \alpha \in \mathbb{R}$, be the translation group in the $y$-direction defined by

$$
\left(U_{\alpha} \psi\right)(y)=\psi(y+\alpha) .
$$

Since the representation is unitary, the operator

$$
A:=-\frac{i}{2}\left(y U_{T}-U_{-T} y\right),
$$

is self-adjoint on the domain $D(y)$ of the operator multiplication by $y$ (since $U_{\alpha}$ preserves this domain) and is equal to the operator $A_{f}$ defined by (3.1) for $f=-i U_{T} \in \mathcal{M}$. The two main reasons for this choice of $f$ are, first, that (3.2) actually holds true for $T$ sufficiently small,

$$
\min _{k \in E_{r}^{-1}\left(\left[E-\delta_{0}, E+\delta_{0}\right]\right)} \sin (T k) E_{r}^{\prime}(k)>0, r=1, \ldots, n, T \in\left(0, \frac{\pi}{E_{1}^{-1}\left(E+\delta_{0}\right)}\right),
$$

where $E_{1}^{-1}$ denotes the function inverse to $E_{1}:[0, \infty) \rightarrow \mathbb{R}$, and, second, the vanishing of the commutator terms $\left[V, U_{ \pm T}\right]=0$, involving

$$
[V, i A]=\frac{1}{2}\left(y\left[V, U_{T}\right]-\left[V, U_{-T}\right] y\right)=0 .
$$

For $E \in \mathbb{R}$ and $\delta>0$ set $\Delta_{E}(\delta):=(E-\delta / 2, E+\delta / 2)$. 
Proposition 3.1. Let $n, E$ and $\delta_{0}$ be as in Lemma 3.1. Assume that (3.5) holds true for some $T \in\left(0, \frac{\pi}{E_{1}^{-1}\left(E+\delta_{0}\right)}\right)$ and let $A=A_{f}$ be defined by $(3.1)$ for $f(k)=-i e^{i T k}, k \in \mathbb{R}$. Let $\delta=\delta(E) \in\left(0, \delta_{0}\right)$ and $\|V\|_{\infty}$ be so small that the condition (3.15) holds true. Then there is a constant $C>0$ such that

$$
\mathbb{P}_{\Delta_{E}(\delta)}[H, i A] \mathbb{P}_{\Delta_{E}(\delta)} \geq C \mathbb{P}_{\Delta_{E}(\delta)}
$$

where the commutator $[H, i A]$ is understood as a bounded operator from $\mathcal{H}_{2}$ to $L^{2}\left(\mathcal{S}_{L}\right)$.

Proof. Taking into account (3.6), straightforward computations yield

$$
[H, i A]=-2 \sin \left(T p_{y}\right)\left(p_{y}-b x\right)
$$

on $D(A) \cap \mathcal{H}_{2}$. Hence $[H, i A]$ extends to a bounded operator from $\mathcal{H}_{2}$ to $L^{2}\left(\mathcal{S}_{L}\right)$.

Further we decompose $\psi \in \mathbb{P}_{\Delta_{E}(\delta)}(H) L^{2}\left(\mathcal{S}_{L}\right)$ as

$$
\psi=\phi+\xi, \phi:=\mathbb{P}_{\Delta_{E}\left(\delta_{0}\right)}\left(H_{0}\right) \psi, \xi:=\mathbb{P}_{\Delta_{E}^{c}\left(\delta_{0}\right)}\left(H_{0}\right) \psi
$$

Choose $\chi$ in Lemma 3.1 to be equal to one on $\Delta_{E}\left(\delta_{0}\right)$, and multiply $(3.3)$ from both sides by $\mathbb{P}_{\Delta_{E}\left(\delta_{0}\right)}\left(H_{0}\right)$. Thus, we obtain

$$
\left\langle\phi,\left[H_{0}, i A\right] \phi\right\rangle \geq C_{E}\left(\delta_{0}\right)\|\phi\|^{2}
$$

Using (3.6)-(3.9), we find that

$$
\langle\psi,[H, i A] \psi\rangle=\left\langle\psi,\left[H_{0}, i A\right] \psi\right\rangle=\left\langle\phi,\left[H_{0}, i A\right] \phi\right\rangle+G(\phi, \xi)
$$

where the perturbation term has the expression

$$
G(\phi, \xi)=\int_{\mathbb{R}} \sin (T k)\langle\hat{\xi}(., k),(k-b x) \hat{\xi}(., k)\rangle d k+2 \operatorname{Re}\left(\int_{\mathbb{R}} \sin (T k)\langle\hat{\phi}(., k),(k-b x) \hat{\xi}(., k)\rangle d k\right),
$$

$\hat{\xi}$ and $\hat{\phi}$ denoting respectively $\mathcal{F} \xi$ and $\mathcal{F} \phi$. By (3.11)-(3.12),

$$
\langle\psi,[H, i A] \psi\rangle \geq\left\langle\phi,\left[H_{0}, i A\right] \phi\right\rangle-2\left\|\left(p_{y}-b x\right) \xi\right\|\|\psi\| .
$$

Moreover the identity $\|\xi\|^{2}=\left\langle(H-E-V) \psi,\left(H_{0}-E\right)^{-1} \xi\right\rangle$ yields

$$
\|\xi\| \leq\left(\frac{\delta+\|V\|_{\infty}}{\delta_{0}}\right)\|\psi\| \text { and }\left\|\left(p_{y}-b x\right) \xi\right\|^{2} \leq\left\langle\xi, H_{0} \xi\right\rangle \leq\left(\mathcal{E}_{n+1}+\|V\|_{\infty}\right)\|\xi\|\|\psi\|,
$$

whence

$$
\langle\psi,[H, i A] \psi\rangle \geq\left[C_{E}\left(\delta_{0}\right)\left(1-\left(\frac{\delta+\|V\|_{\infty}}{\delta_{0}}\right)^{2}\right)-2\left(\mathcal{E}_{n+1}+\|V\|_{\infty}\right)^{1 / 2}\left(\frac{\delta+\|V\|_{\infty}}{\delta_{0}}\right)^{1 / 2}\right]\|\psi\|^{2}
$$

by combining (3.10) with (3.13)-(3.14). The result follows from this provided $\delta$ and $\|V\|_{\infty}$ are taken small enough so that

$$
\left(\frac{\delta+\|V\|_{\infty}}{\delta_{0}}\right)^{2}+2 C_{E}\left(\delta_{0}\right)^{-1}\left(\mathcal{E}_{n+1}+\|V\|_{\infty}\right)^{1 / 2}\left(\frac{\delta+\|V\|_{\infty}}{\delta_{0}}\right)^{1 / 2}<1
$$

Notice from (3.8) that $[[H, i A], i A]=0$ by direct calculation (see [16, Section 1.3]). Since $[H, i A]$ extends to a bounded operator from $\mathcal{H}_{2}$ into $L^{2}\left(\mathcal{S}_{L}\right)$, and the double commutator of $H$ with $A$ vanishes, the Mourre estimate (3.7) combined with [5, Corollary 4.10] entail the following 
Corollary 3.1. Let $n, E, \delta_{0}$ and $T$ be as in Proposition 3.1. If $\delta$ and $\|V\|_{\infty}$ satisfy (3.15), then the spectrum of $H$ in $\Delta_{E}(\delta)$ is absolutely continuous.

Proposition 3.1 and Corollary 3.1 are similar to [17, Proposition 3.3] and the results of [16, Section 1.3], obtained for a slightly different model defined in Section 4.

Theorem 3.2. Let $\Delta$ be a compact subinterval of $\left(\mathcal{E}_{n}, \mathcal{E}_{n+1}\right), n \in \mathbb{N}$. If $T$ and $\|V\|_{\infty}$ are sufficiently small, then the spectrum of $H$ in $\Delta$ is absolutely continuous.

Proof. For $E_{+}:=\sup _{E \in \Delta}\left(E+\delta_{0}(E)\right) \in\left(\mathcal{E}_{n}, \mathcal{E}_{n+1}\right]$, choose $T \in\left(0, \frac{\pi}{E_{1}^{-1}\left(E_{+}\right)}\right)$. Evidently

$$
T \in\left(0, \frac{\pi}{E_{1}^{-1}\left(E+\delta_{0}\right)}\right), E \in \Delta,
$$

by (2.3). Assume $\Delta \subset\left(\mathcal{E}_{n}, \mathcal{E}_{n+1}\right)$. For all $E \in \Delta$, choose $\delta(E) \in\left(0, \delta_{0}(E)\right)$ and $v_{E}>0$ such that

$$
F_{n, E}\left(v_{E}\right):=\left(\frac{\delta(E)+v_{E}}{\delta_{0}(E)}\right)^{2}+2 C_{E}\left(\delta_{0}(E)\right)^{-1}\left(\mathcal{E}_{n+1}+v_{E}\right)^{1 / 2}\left(\frac{\delta(E)+v_{E}}{\delta_{0}(E)}\right)^{1 / 2}<1
$$

Since $\Delta$ is compact and $\Delta \subset \cup_{E \in \Delta} \Delta_{E}(\delta(E))$ there exists a finite set $\left\{E_{j}\right\}_{j=1}^{N}$ of energies in $E_{j} \in \Delta$ such that

$$
\Delta \subset \cup_{j=1}^{N} \Delta_{E_{j}}\left(\delta\left(E_{j}\right)\right)
$$

Assume that $\|V\|_{\infty} \in\left(0, \min _{j=1 \ldots, N} v_{E_{j}}\right)$. Since $v \mapsto F_{n, E_{j}}(v), j=1, \ldots, N$, is increasing, we have $F_{n, E_{j}}\left(\|V\|_{\infty}\right)<1$, by (3.17). This, combined (3.16) and Corollary 3.1, shows that the spectrum of $H$ is absolutely continuous in $\Delta_{E_{j}}\left(\delta\left(E_{j}\right)\right)$ for $j=1, \ldots, N$. Hence the result follows from (3.18).

3.4. In this subsection we address the case of an electric potential $V: \mathcal{S}_{L} \rightarrow \mathbb{R}$ decaying in the $y$-direction in the sense that

$$
V H_{0}^{-1} \in S_{\infty}
$$

and

$$
H_{0}^{-1} y \frac{\partial V}{\partial_{y}} H_{0}^{-1} \in S_{\infty}
$$

Due to (3.19) the operator $H=H_{0}+V$ is self-adjoint on the domain of $H_{0}$, and we have

$$
\sigma_{\text {ess }}(H)=\sigma_{\text {ess }}\left(H_{0}\right)=\left[\mathcal{E}_{1},+\infty\right) .
$$

Proposition 3.2. ([2, Proposition 3.1]) Assume (3.19) - (3.20). Let n, E, $\delta_{0}$ and $\chi$ be as in Lemma 3.1. Let $A=A_{f}$ be defined by (3.1) for $f(k)=k, k \in \mathbb{R}$. Then there exists a constant $C>0$ such that

$$
\mathbb{P}_{\Delta_{E}\left(\delta_{0}\right)}(H)[H, i A] \mathbb{P}_{\Delta_{E}\left(\delta_{0}\right)}(H) \geq C \mathbb{P}_{\Delta_{E}\left(\delta_{0}\right)}(H)+K
$$

where the commutator $[H, i A]$ is understood as a bounded operator from $\mathcal{H}_{2}$ into $\mathcal{H}_{-2}$ and $K \in S_{\infty}$.

Proof. We have

$$
[H, i A]=\left[H_{0}, i A\right]+[V, i A],\left[H_{0}, i A\right]=2 p_{y}^{2}-2 b x p_{y}, \quad[V, i A]=-y \frac{\partial}{\partial V}(x, y),
$$

by direct computations. Thus $[H, i A]$ is a bounded operator from $\mathcal{H}_{2}$ into $\mathcal{H}_{-2}$ since this is the case for $\left[H_{0}, i A\right]$ and $H_{0}[V, i A] H_{0}^{-1}$ is compact by $(3.20)$.

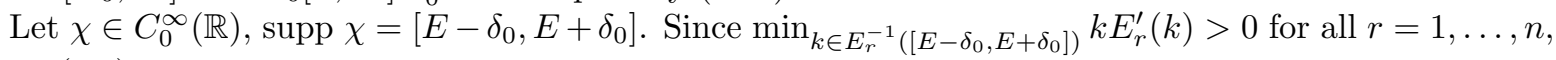
by $(2.3)$, Lemma 3.1 yields

$$
\chi\left(H_{0}\right)\left[H_{0}, i A\right] \chi\left(H_{0}\right) \geq C \chi\left(H_{0}\right)^{2} .
$$


Further by (3.22),

$$
\chi(H)[H, i A] \chi(H)=\chi\left(H_{0}\right)\left[H_{0}, i A\right] \chi\left(H_{0}\right)+K_{0},
$$

where $K_{0}:=K_{1}+K_{2}-K_{3}$ and

$$
K_{1}:=\chi\left(H_{0}\right)\left[H_{0}, i A\right]\left(\chi(H)-\chi\left(H_{0}\right)\right), K_{2}:=\left(\chi(H)-\chi\left(H_{0}\right)\right)\left[H_{0}, i A\right] \chi(H), K_{3}:=\chi(H) y \frac{\partial V}{\partial_{y}} \chi(H) .
$$

Assuming (3.19) we have $\chi(H)-\chi\left(H_{0}\right) \in S_{\infty}$ by [2, Lemma 3.2]. Hence the operator

$K_{1}=\chi\left(H_{0}\right) H_{0} H_{0}^{-1}\left[H_{0}, i A\right]\left(\chi(H)-\chi\left(H_{0}\right)\right)$ is compact since $\chi\left(H_{0}\right) H_{0}$ and $H_{0}^{-1}\left[H_{0}, i A\right]$ extend to bounded operators in $L^{2}\left(\mathcal{S}_{L}\right)$. Similarly, $\left[H_{0}, i A\right] H_{0}^{-1}$ and $H_{0} \chi(H)=H \chi(H)-V \chi(H)$ being bounded, $K_{2}=\left(\chi(H)-\chi\left(H_{0}\right)\right)\left[H_{0}, i A\right] H_{0}^{-1} H_{0} \chi(H)$ is compact. Further (3.20) entails that

$K_{3}=\chi(H) y \frac{\partial V}{\partial y} \chi(H)=\chi(H) H_{0} H_{0}^{-1} y \frac{\partial V}{\partial y} H_{0}^{-1} H_{0} \chi(H)$ is compact too. Therefore, $K_{0}=K_{1}+K_{2}-K_{3} \in$ $S_{\infty}$. Combining (3.23) and (3.24), we get

$$
\chi(H)[H, i A] \chi(H) \geq C \chi\left(H_{0}\right)^{2}+K_{0}=C \chi(H)^{2}+K_{0}+K_{4},
$$

where $K_{4}:=C\left(\chi\left(H_{0}\right)^{2}-\chi(H)^{2}\right) \in S_{\infty}$ by substituting $\chi^{2}$ for $\chi$ in [2, Lemma 3.2].

Finally, we obtain $(3.21)$ with $K=\mathbb{P}_{\Delta_{E}\left(\delta_{0}\right)}(H)\left(K_{0}+K_{4}\right) \mathbb{P}_{\Delta_{E}\left(\delta_{0}\right)}(H) \in S_{\infty}$ upon choosing $\chi$ in (3.25) to be equal to one on $\Delta_{E}\left(\delta_{0}\right)$, and multiplying (3.25) from both sides by $\mathbb{P}_{\Delta_{E}\left(\delta_{0}\right)}(H)$.

Theorem 3.3. ([2, Theorem 3.1]) (i) Assume (3.19)-(3.20). Then $H$ has at most finitely many eigenvalues in any compact subinterval of $\mathbb{R} \backslash \mathcal{Z}$, and each eigenvalue has finite multiplicity.

(ii) Suppose moreover

$$
\begin{aligned}
& H_{0}^{-1 / 2} y \frac{\partial V}{\partial y} H_{0}^{-1} \in \mathcal{B}, \\
& H_{0}^{-1} y^{2} \frac{\partial^{2} V}{\partial y^{2}} H_{0}^{-1} \in \mathcal{B} .
\end{aligned}
$$

Then $\sigma_{\mathrm{sc}}(H)=\emptyset$.

Proof. Let $\Delta \subset \mathbb{R} \backslash \mathcal{Z}$ be a compact interval. If $\Delta \subset\left(-\infty, \mathcal{E}_{1}\right)$, then $\Delta \cap \sigma_{\text {ess }}(H)=\emptyset \operatorname{since} \inf \sigma_{\text {ess }}(H)=$ $\mathcal{E}_{1}$, and (i) holds true. Assume $\Delta \subset\left(\mathcal{E}_{n}, \mathcal{E}_{n+1}\right), n \in \mathbb{N}$. For each $E \in \Delta$ choose $\delta_{0}=\delta_{0}(E)$ as in Proposition 3.2. Then we have $\Delta \subset \cup_{E \in \Delta} \Delta_{E}\left(\delta_{0}\right)$. Since $\Delta$ is compact, there exists a finite set $\left\{E_{j}\right\}_{j=1}^{N}$ of energies $E_{j} \in \Delta$ such that

$$
\Delta \subset \cup_{j=1}^{N} \Delta_{E_{j}}\left(\delta_{0}\right) .
$$

The set $D(A) \cap \mathcal{H}_{2}$ being dense in $\mathcal{H}_{2}$ and $[H, i A]$ being a bounded operator from $\mathcal{H}_{2}$ into $\mathcal{H}_{-2}$, the Mourre estimate (3.21) for the energy $E_{j}, j=1, \ldots, N$, combined with the results of [21], [5, Theorem 4.7] and [14] entail that $H$ has at most finitely many eigenvalues in $\Delta_{E_{j}}\left(\delta_{0}\right)$, each eigenvalue having a finite multiplicity. This together with (3.28), proves the first part of Theorem 3.3.

Fix $E \in\left(\mathcal{E}_{n}, \mathcal{E}_{n+1}\right) \backslash \overline{\sigma_{p}(H)}, n \in \mathbb{N}$, and choose $\delta_{0}=\delta_{0}(E)$ as in Proposition 3.2. Then, arguing as in the proof of [5, Lemma 4.8] and [2, Corollary 3.2], it follows from (3.21) that

$$
\mathbb{P}_{\Delta_{E}(\delta)}(H)[H, i A] \mathbb{P}_{\Delta_{E}(\delta)}(H) \geq \frac{C}{2} \mathbb{P}_{\Delta_{E}(\delta)}(H)
$$

for $\delta \in\left(0, \delta_{0}\right)$ sufficiently small, the constant $C$ being same as in (3.21). Assume moreover (3.26) - (3.27) so $[H, i A]$ extends to a bounded operator from $\mathcal{H}_{2}$ to $\mathcal{H}_{-1}$, and the second commutator $[[H, i A], i A]$ extends to a bounded operator from $\mathcal{H}_{2}$ to $\mathcal{H}_{-2}$. Then we obtain $\sigma_{\text {sc }}(H) \cap\left(\left(\mathcal{E}_{n}, \mathcal{E}_{n+1}\right) \backslash \overline{\sigma_{p}(H)}\right)=\emptyset, n \in$ $\mathbb{N}$, by combining (3.29) with the results of $\left[5\right.$, Corollary 4.10] and [14], and hence $\sigma_{\text {sc }}(H) \cap\left(\mathcal{E}_{n}, \mathcal{E}_{n+1}\right)=\emptyset$, $n \in \mathbb{N}$, since the set $\left(\mathcal{E}_{n}, \mathcal{E}_{n+1}\right) \cap \overline{\sigma_{p}(H)}$ is at most discrete. Finally, since $\mathcal{E}_{1}=\inf \sigma_{\text {ess }}(H)$ we have $\sigma_{\mathrm{sc}}(H) \cap\left(-\infty, \mathcal{E}_{1}\right)=\emptyset$. Therefore, $\sigma_{\mathrm{sc}}(H) \cap(\mathbb{R} \backslash \mathcal{Z})=\emptyset$, proving the second part of Theorem 3.3 since $\mathcal{Z}$ is discrete. 


\section{Related models}

4.1. In the physical literature (see e.g. $[22,23,24,25])$ the Dirichlet boundary conditions at $x= \pm L$, are sometimes modeled by introducing an appropriate confining potential $V_{L}$ supported in the complement set of $(-L, L) \times \mathbb{R}$. Among them the "square well model" corresponding to a potential barrier of the form $V_{L}(x):=1-\chi_{(-L, L)}(x)$, where $\chi_{(-L, L)}$ denotes the characteristic function of the interval $(-L, L)$, is an idealization of the edge of an abrupt non-uniform electric potential (see $[4,16])$. In the remaining of this section we address the case of this particular model although most of the results described below generalize for a wide class of more general confining potentials (see [16]).

4.2. In this subsection we introduce the Schrödinger operator considered in this model and review some of its spectral properties that are needed in Section 4.3. Due to the translational invariance in the $y$-direction, the 2D Schrödinger operator

$$
H_{0, g}:=p_{x}^{2}+\left(p_{y}-b x\right)^{2}+g V_{L}(x), g>0,
$$

defined on the dense domain $C_{0}^{\infty}\left(\mathbb{R}^{2}\right)$ and extended to a self-adjoint operator in $L^{2}\left(\mathbb{R}^{2}\right)$, admits a fibre decomposition similar to $(2.1)$. The associated fibred operators $\hat{H}_{0, g}(k)$ have discrete and simple spectrum which equals the increasing sequence $\left\{E_{j, g}(k)\right\}_{k=1}^{\infty}$ of the corresponding eigenvalues. For all $j \in \mathbb{N}$ and $g>0, E_{j, g}$ is an even real analytic function satisfying

$$
(2 j-1) b \leq E_{j, g}(k)<(2 j-1) b+g, k \in \mathbb{R},
$$

and

$$
\lim _{k \rightarrow+\infty} E_{j, g}(k)=(2 j-1) b+g .
$$

Hence $\sigma\left(H_{0, g}\right)=\sigma_{\mathrm{ac}}\left(H_{0, g}\right)=\cup_{j=1}^{\infty}\left[\mathcal{E}_{j, g},(2 j-1) b+g\right]$, where $\mathcal{E}_{j, g}:=\inf _{k \in \mathbb{R}} E_{j, g}(k) \in[(2 j-1) b,(2 j-$ $1) b+g)$. Note that if $g<2 b$ there are nontrivial open gaps $\left((2 j-1) b+g, \mathcal{E}_{j+1, g}\right), j \in \mathbb{N}$, in $\sigma\left(H_{0, g}\right)$. By [16, Lemma 5.3] there are two constants $\alpha_{j}>0$ and $\mu_{j}>0$ independent of $g, b$ and $L$, such that we have

$$
0 \leq \frac{\mathcal{E}_{j, g}-(2 j+1) b}{b} \leq \alpha_{j} e^{-\mu_{j} b L^{2}}, j \in \mathbb{N}
$$

for $b L^{2}>1$. Thus upon choosing $b L^{2}$ sufficiently large, a set of the form

$$
\Delta_{n}=\Delta_{n}(a, c):=[(2 n+a) b,(2 n+c) b], 1<a<c<3, n \in \mathbb{N},
$$

is contained in $\left(\mathcal{E}_{n}, \mathcal{E}_{n+1}\right)$.

For all $j \in \mathbb{N}$ and $k \in \mathbb{R}, E_{j, g}(k)$ is easily seen to be an increasing function of $g$. This follows from the Feynmann-Hellman formula

$$
\partial_{g} E_{j, g}(k)=\left\langle V_{L} \psi_{j, g}(. ; k), \psi_{j, g}(. ; k)\right\rangle>0,
$$

where $\psi_{j, g}(. ; k): \mathbb{R} \rightarrow \mathbb{R}$ is the real-valued normalized in $L^{2}(\mathbb{R})$ eigenfunction of the operator $\hat{H}_{0, g}(k)$ associated to $E_{j, g}(k)$. Moreover, in view of [16, Lemma 3.3] the "Dirichlet boundary condition model" can be seen as the limit model as $g$ goes to infinity of the "square well model", in the sense that for $\Delta_{n}, n \in \mathbb{N}$, as in (4.30), and $b L^{2}$ sufficiently large, there is a constant $\zeta_{n}>0$ independent of $g$, such that we have

$$
0 \leq E_{j}(k)-E_{j, g}(k) \leq \zeta_{n} g^{-1 / 2}, k \in E_{j, g}^{-1}\left(\Delta_{n}\right), j=1,2, \ldots, n, g \geq(2 n+3) b,
$$

and

$$
\left\|\mathbb{P}_{\Delta_{n}}\left(\hat{H}_{0}(k)\right)-\mathbb{P}_{\Delta_{n}}\left(\hat{H}_{0, g}(k)\right)\right\| \leq \zeta_{n} g^{-1 / 2}, k \in E_{j, g}^{-1}\left(\Delta_{n}\right), j=1,2, \ldots, n, g \geq(2 n+3) b,
$$


the norm being taken in $L^{2}\left(\mathcal{S}_{L}\right)$.

Further,

$$
E_{j, g}^{\prime}(k)=2 \int_{\mathbb{R}}(k-b x) \psi_{j, g}(k)^{2} d x, j \in \mathbb{N}, k \in \mathbb{R},
$$

by the Feynman-Hellmann Theorem. Taking into account the eigenvalue equation $\hat{H}_{0, g}(k) \psi_{j, g}(k)=$ $E_{j, g}(k) \psi_{j, g}(k)$ and the identity $\partial_{k}(k-b x)^{2}=-b^{-1} \partial_{x}(k-b x)^{2}$, an integration by parts in (4.31) yields

$$
E_{j, g}^{\prime}(k)=\frac{g}{b}\left(\psi_{j, g}(L ; k)^{2}-\psi_{j, g}(-L ; k)^{2}\right) .
$$

Consequently the sign of the derivative $E_{j, g}^{\prime}$ is determined by the trace of the corresponding eigenfunction along the two boundary components at $x= \pm L$. Let $\Delta_{n}, n \in \mathbb{N}$, be as in (4.30). Since the domain $x \approx-L$ is in the classically forbidden region for energies $E_{j, g}(k)$, for $j=1,2, \ldots, n$ and $k \in E_{j, g}^{-1}\left(\Delta_{n}\right) \cap(0,+\infty)$, the contribution $\psi_{j, g}^{2}(-L ; k)^{2}$ will be exponentially small relative to $\psi_{j, g}(L ; k)^{2}$. Namely, by [16, Lemma 2.2] there are two constants $C_{n}>0$ and $\gamma_{n}>0$, depending only on $n, a$ and $c$, such that

$$
\min _{k \in E_{j, g}^{-1}\left(\Delta_{n}\right) \cap(0,+\infty)} E_{j, g}^{\prime}(k) \geq C_{n} b^{1 / 2}, j=1, \ldots, n,
$$

provided $g \geq(2 n+3) b$ and $b L^{2} \geq \gamma_{n}$.

Finally the $E_{j, g}$ being simple and continuous, a statement analogous to Lemma 2.1 holds true for the "square well model" (see [16, Lemma 2.1]): there is $\delta_{n, g}>0$ such that any interval $\Delta \subset\left(\mathcal{E}_{n, g}, \mathcal{E}_{n+1, g}\right.$ ) with $|\Delta|<\delta_{n, g}$, satisfies $E_{r, g}^{-1}(\Delta)=\emptyset$ for $r \geq n+1$ and $E_{r, g}^{-1}(\Delta) \cap E_{s, g}^{-1}(\Delta)=\emptyset$ for $r \neq s, r, s=1, \ldots, n$.

4.3. Let $\Delta_{n}, n \in \mathbb{N}$, be defined by (4.30). In this subsection we perturb $H_{0, g}$ by a suitable potential $V$ and outline that a Mourre estimate for $H_{g}:=H_{0, g}+V$ can be deduced from (4.32) on any sufficiently small subinterval of $\Delta_{n}$, under convenient assumptions on $b, L$ and $g$. To make this statement more precise, introduce the operator

$$
A=-\frac{i}{2}\left(y U_{\alpha}-U_{-\alpha} y\right), \alpha=\frac{\pi}{2 b L},
$$

where $U_{\alpha}=e^{i \alpha p_{y}}$, as in Section 3.3. By straightforward computations,

$$
\left[H_{0, g}, i A\right]=\sin \left(\frac{\pi}{2 b L} p_{y}\right)\left(p_{y}-b x\right)
$$

as quadratic forms on $D(A) \cap D\left(H_{0, g}\right)$. In light of (4.33), the main reason for this choice of $A$ is given by [16, Lemma 5.8]: for all $g \geq(2 n+3) b$ and $b L^{2} \geq \gamma_{n}, \gamma_{n}$ being the same as in (4.32), we have

$$
|k-b L| \leq \kappa_{n} b^{1 / 2}, k \in E_{j, g}^{-1}\left(\Delta_{n}\right) \cap(0,+\infty), j=1, \ldots, n,
$$

for some constant $\kappa_{n}>0$ depending only on $n, a$ and $c$. Indeed, $L$ being fixed in $(0,+\infty)$, it follows from this and from (4.32) that there is a constant $\beta_{n}>0$ depending only on $n, a, c$ and $L$, such that we have

$$
\min _{k \in E_{j, g}^{-1}\left(\Delta_{n}\right) \cap(0,+\infty)} \sin \left(\frac{\pi}{2 b L} k\right) E_{j, g}^{\prime}(k) \geq\left(C_{n} / 2\right) b^{1 / 2}, j=1, \ldots, n,
$$

for all $g \geq(2 n+3) b$ and $b \geq \beta_{n}$.

Proposition 4.1. ([17, Proposition 3.4]) Let $\Delta_{n}, n \in \mathbb{N}$, be as in (4.30) and $\Delta_{E}(\delta)=(E-\delta / 2, E+\delta /$ 2) be contained in $\Delta_{n}$. Fix $L>0$ and $b \geq \beta_{n}$, where $\beta_{n}$ is as in (4.34). Let $A=A_{f}$ be defined by (3.1) for $f(k)=i e^{i \frac{\pi}{2 b L} k}, k \in \mathbb{R}$, and assume that

$$
V, y V \in L^{\infty}\left(\mathbb{R}^{2}\right) .
$$


Then for all $g \geq(2 n+3) b$ there is a constant $C(\delta)>0$, independent of $E$, such that

$$
\mathbb{P}_{H_{g}}\left(\Delta_{E}(\delta)\right)\left[H_{g}, i A\right] \mathbb{P}_{H_{g}}\left(\Delta_{E}(\delta)\right) \geq C(\delta) b^{1 / 2} \mathbb{P}_{H_{g}}\left(\Delta_{E}(\delta)\right)
$$

provided $\delta,\|V\|_{\infty}$ and $\|y V\|_{\infty}$ are taken so small that (4.39) holds true.

Proof. Fix $b \geq \beta_{n}, g \geq(2 n+3) b$ and $\delta \in\left(0, \delta_{n, g}\right)$. Due to (4.33)-(4.34) we get

$$
\mathbb{P}_{H_{0, g}}\left(\Delta_{E}(\delta)\right)\left[H_{0, g}, i A\right] \mathbb{P}_{H_{0, g}}\left(\Delta_{E}(\delta)\right) \geq\left(C_{n} / 2\right) b^{1 / 2} \mathbb{P}_{H_{0, g}}\left(\Delta_{E}(\delta)\right),
$$

by arguing as in the derivation of Lemma 3.1. Further, taking into account (4.35), we find

$$
|\langle\psi,[V, i A] \psi\rangle| \leq\left(2\|y V\|_{\infty}+\frac{\pi}{2 b L}\|V\|_{\infty}\right)\|\psi\|^{2}, \psi \in D(A),
$$

by straightforward computations. Thus, by repeating the arguments of the proof of Proposition 3.1, we obtain that (4.37)-(4.38) entail (4.36) provided $\delta,\|V\|_{\infty}$ and $\|y V\|_{\infty}$ are taken so small that

$$
\left(\frac{\delta+\|V\|_{\infty}}{\delta_{n, g}}\right)^{2}+4 C_{n}^{-1} b^{-1 / 2}\left[\left(\mathcal{E}_{n+1}+\|V\|_{\infty}\right)^{1 / 2}\left(\frac{\delta+\|V\|_{\infty}}{\delta_{n, g}}\right)^{1 / 2}+2\|y V\|_{\infty}+\frac{\pi}{2 b L}\|V\|_{\infty}\right]<1
$$

Notice that the double commutator $[[H, i A], i A]=[[V, i A], i A]$ extends to a bounded operator on $L^{2}\left(\mathbb{R}^{2}\right)$ if $y^{2} V \in L^{\infty}\left(\mathbb{R}^{2}\right)$. Hence, by mimicking the proof of Theorem 3.2, we obtain the following

Theorem 4.1. Let $\Delta_{n}, n \in \mathbb{N}$, be as in (4.30). Fix $L>0$ and $g \geq(2 n+3) b$. Assume (4.35) and $y^{2} V \in L^{\infty}\left(\mathbb{R}^{2}\right)$. Then for all $b$ sufficiently large the spectrum of $H$ in $\Delta_{n}$ is absolutely continuous provided $\|V\|_{\infty}$ and $\|y V\|_{\infty}$ are small enough.

Remark 4.2. (i) Notice in particular that if $b \gg 1$ and $\delta_{n, g}$ is $O(b)$, then (4.36)-(4.39) entail a Mourre estimate for $H_{g}$ on $\Delta_{E}(\delta)$ for perturbations of the size of $b$.

(ii) On the other hand, by assuming

$$
\frac{\partial V}{\partial y}, y \frac{\partial V}{\partial y} \in L^{\infty}\left(\mathbb{R}^{2}\right)
$$

in Proposition 4.1, instead of (4.35), it is not hard to check that (4.38) can be replaced by

$$
|\langle\psi,[V, i A] \psi\rangle| \leq\left(\left(\frac{\pi}{2 b L}\right)^{2}\left\|\frac{\partial V}{\partial y}\right\|_{\infty}+\frac{\pi}{2 b L}\left\|y \frac{\partial V}{\partial y}\right\|_{\infty}\right)\|\psi\|^{2}, \psi \in D(A) .
$$

Thus, under the conditions specified in (i), the same conclusion holds true for perturbations $V$ of size $O\left(b^{2}\right)$.

Acknowledgements. (i) P. D. Hislop was partially supported by NSF grant 0503784 .

(ii) G. Raikov was partially supported by the Chilean Science Foundation Fondecyt under Grant 1050716, and by Núcleo Científico ICM P07-027-F "Mathematical Theory of Quantum and Classical Magnetic Systems".

(iii) E. Soccorsi wants to thank the organizers of the conference on Spectral and Scattering Theory for Quantum Magnetic Systems, CIRM, Luminy, July 2008, for inviting him to present this topic. 


\section{References}

[1] J. Bellissard, A. van Elst, H. Schulz-Baldes, The noncommutative geometry of the quantum Hall effect, J. Math. Phys. 35 (1994), 5373-5451.

[2] P. Briet, G. Raikov, E. Soccorsi, Spectral Properties of a Magnetic Quantum Hamiltonian on a Strip, Asymptotic Analysis 58 (2008), 127-155.

[3] J.-M. Combes, F. Germinet, Stability of the edge conductivity in quantum Hall systems, Commun. Math. Phys. 256 (2005), 159-180.

[4] J.-M. Combes, P. D. Hislop, E. Soccorsi, Edge states for quantum Hall systems, Cont. Math. 307 (2002), 69-81.

[5] H. Cycon, R. Froese, W. Kirsch, B. Simon, Schrödinger Operators with Application to Quantum Mechanics and Global Geometry, Texts and Monographs in Physics, Springer-Verlag, Berlin, Heidelberg, New York, 1987.

[6] S. De Bièvre, J. V. Pulé, Propagating edge states for a magnetic Hamiltonian, Math. Phys. Electron. J. 5 (1999), Paper 3, 17 pp.

[7] P. Elbau, G.M. Graf, Equality of bulk and edge Hall conductance revisited, Comm. Math. Phys. 229 no. 3 (2002), 415-432.

[8] P. Exner, A. Joye, H. Kovarik, Magnetic transport in a straight parabolic channel, J. Phys. A 34 (2001), 9733-9752.

[9] C. Ferrari, N. Macris, Spectral properties of finite quantum Hall systems, Operator algebras and mathematical physics (Constanţa 2001), Theta, Bucharest (2003), 115-122.

[10] C. Ferrari, N. Macris, Intermixture of extended edge and localized bulk energy levels in macroscopic Hall systems, J. Phys. A: Math. Gen. A 35 (2002), 6339-6358.

[11] C. Ferrari, N. Macris, Extended edge states in finite Hall systems, J. Math. Phys. 44 no. 9 (2003), 3734-3751.

[12] J. Fröhlich, G. M. Graf, J. Walcher, On the extended nature of edge states of quantum Hall Hamiltonians, Ann. H. Poincaré 1 (2000), 405-444.

[13] V. GelLer, M. Senatorov, The structure of the spectrum of the Schrödinger operator with a magnetic field in a strip, and finite-gap potentials, Mat. Sb. 188 (1997), 21-32 (Russian); English translation in Sb. Math. 188 (1997), 657-669.

[14] V. Georgescu, C. Gérard, On the virial theorem in quantum mechanics, Comm. Math. Phys. 208 (1999), 275-281.

[15] P. D. Hislop, E. Soccorsi, Edge Currents for Quantum Hall Systems, I. One-Edge, Unbounded Geometries, Reviews in Math. Phys. 20 (2008), 71-115.

[16] P. D. Hislop, E. Soccorsi, Edge Currents for Quantum Hall Systems, II. Two-Edge, Bounded and Unbounded Geometries, Ann. H. Poincaré 9 (2008), 1141-1171.

[17] P. D. Hislop, E. Soccorsi, Edge Currents for Quantum Hall Systems, II. Two-Edge, Bounded and Unbounded Geometries, http://arXiv.org/math-ph/0702093, archived.

[18] T. Kato, Perturbation Theory for Linear Operators, Die Grundlehren der mathematischen Wissenschaften, 132 Springer-Verlag New York, Inc., New York, 1966. 
[19] J. Kellendonk, T. Richter, H. Schulz-Baldes, Edge channels and Chern numbers in the integer quantum Hall effect, Rev. Math. Phys. 14 (2002), 87-119.

[20] N. Macris, P. A. Martin, J. V. Pulé, On edge states in semi-infinite quantum Hall systems, J. Phys. : Math. Gen A 32 (1999), 1985-1996.

[21] E. Mourre, Absence of singular continuous spectrum for certain self-adjoint operators, Comm. Math. Phys. 78 (1981), 391-408.

[22] J.-P. Muscat, Response function for a finite step potential model, Surface Science $\mathbf{5 8}$ vol. 2 (1976), 557-565.

[23] J. Villavicencio, Exact relativistic time evolution for a step potential barrier, J. Phys. A : Math. Gen. 33 (2000), 6061-6072.

[24] J. Villavicencio, R. Romo, S.S. Silva, Quantum wave evolution in a step potential barrier, J. Phys. A : Math. Gen. 66 (2002), 042110.

[25] V.M. Zhilin, S.V. IordanskiI, Scattering of electrons by a potential step in a magnetic field, J. of Exp. Theo. Phys. 85 (1997), 959-962. 